\title{
USING A GENERALIZED LINEAR MIXED MODEL APPROACH TO EXPLORE THE ROLE OF AGE, MOTOR PROFICIENCY, AND COGNITIVE STYLES IN CHILDREN'S REACH ESTIMATION ACCURACY ${ }^{1}$
}

\author{
PRISCILA M. CAÇOLA \\ Department of Kinesiology
}

MOHAN D. PANT

Department of Curriculum \& Instruction

University of Texas at Arlington

\begin{abstract}
Summary.-The purpose was to use a multi-level statistical technique to analyze how children's age, motor proficiency, and cognitive styles interact to affect accuracy on reach estimation tasks via Motor Imagery and Visual Imagery. Results from the Generalized Linear Mixed Model analysis (GLMM) indicated that only the 7-year-old age group had significant random intercepts for both tasks. Motor proficiency predicted accuracy in reach tasks, and cognitive styles (object scale) predicted accuracy in the motor imagery task. GLMM analysis is suitable to explore age and other parameters of development. In this case, it allowed an assessment of motor proficiency interacting with age to shape how children represent, plan, and act on the environment.
\end{abstract}

The unfolding of motor development across the lifespan has been widely studied over the last decades. Motor development plays a central role in infancy and childhood - in fact, it has been suggested that motor development is the "key" forecaster to the development of certain abilities (Adolph, Tamis-LeMonda, \& Karasik, 2010). For example, Campos, Anderson, Barbu-Roth, Hubbard, Hertenstein, and Witherington (2000) posited that the onset of locomotion is a setting event that changes the social and nonsocial world for infants. Age or maturational state, previously considered the most important triggers for motor changes (e.g., Roberton, 1982), has now taken another role in contemporary views of development. Currently, a fundamental assumption in motor development is that development is age-related, but not age-dependent (Clark \& Metcalfe, 2002; Haywood \& Getchell, 2009; Gallahue, Ozmun, \& Goodway, 2012).

Of course, age is still considered a general indicator of developmental readiness in certain events, e.g., when determining "typical" growth at the pediatrician's office or when determining readiness to enter a grade school. In research studies, however, it is difficult to distinguish the effects of age and other parameters in children's development. In the field of motor development, the most commonly used statistical analyses look at age and other parameters as if they had the same effects on a child's status.

${ }^{1}$ Address correspondence to Priscila Caçola (cacola@uta.edu). 
Therefore, the purpose of this study is to explore an unusual analysis in the field of motor development, the Generalized Linear Mixed Model (GLMM) technique. The GLMM is a multi-level statistical approach that can account for the assumption of "age-related, but not age-dependent" by considering age as a higher level variable and analyzing how other factors of a child's developmental status (in this case, motor proficiency and cognitive styles) interact with age to affect accuracy of reach estimation tasks. With this statistical technique, researchers can explore factors that go beyond chronological age and how these factors interact with age to determine developmental progress.

Whether performing motor skills for daily living or leisure activity, estimations of reach distance are essential for navigating the environment (Caçola, Spessato, \& Gabbard, 2014). Gabbard, Cordova, and Ammar (2007) compared reach estimates of children between five and 11 years of age and young adults and found a significant distinction in reference to peripersonal [within reach] and extrapersonal [beyond reach] space. For children, significantly more error was exhibited with extrapersonal compared to peripersonal space, revealing an overestimation bias in children when compared to adults. It is likely that from that age to early adulthood, individuals use opportunities to refine accuracy and precision with estimating reach and are more able to judge distances from all frames of reference (Caçola, et al., 2014).

Accurate reach estimations are a result of a (proper) combination of factors (e.g., spatial perception, cognitive ability, mental imagery, etc.). In this study, the focus is children's ability to use and combine certain aspects of mental imagery to accurately estimate their reach capacity. Mental imagery underscores memory, reasoning, and learning, and all imagery allows generation of specific predictions based upon past experience in regard to the imminent or distant future (Oliver, Gabbard, Caçola, \& Bobbio, 2012). Two specific aspects of mental imagery are motor imagery and visual imagery. Motor imagery, also known as kinesthetic imagery, is an active cognitive process during which the representation of a specific action is internally reproduced in working memory without any overt motor output (Decety \& Grèzes, 1999). With regards to motor imagery, research suggests that children as young as five years of age have the ability to imagine movements (Funk, Brugger, \& Wilkening, 2005), and such ability appears to be still emerging at seven years of age (Molina, Tijus, \& Jouen, 2008; Frick, Daum, Wilson, \& Wilkening, 2009). While motor imagery involves a representation of the body in action, visual imagery does not involve a motor component but only a simulation linked to the visual perception system (Kosslyn, 1999). In reference to visual imagery, it appears that it can be generated by children as young as two or three years 
of age (e.g., Rieser, Garing, \& Young, 1994), and there are suggestions that children as young as five years of age are capable of more complex forms of mental imagery (Funk, et al., 2005; Caeyenberghs, Tsoupas, Wilson, \& Smits-Engelsman, 2009).

The development of reach estimation abilities can theoretically also be related to developmental aspects of cognitive styles. Cognitive style traditionally refers to consistencies in an individual's manner of cognitive functioning, particularly in information processing and acquisition (e.g., Ausburn \& Ausburn, 1978). In other words, cognitive styles can be viewed as individual preferences when using cognitive abilities. Currently, two dimensions of cognitive styles are related to perception of metric environments. The first is visual-spatial style, a widely accepted component of visual intelligence (Blazhenkova \& Kozhevnikov, 2010), representing a number of related subcomponents (e.g., spatial visualization, spatial relations, etc.) that relate to how individuals deal with materials presented in space, or with how individuals orient themselves in space (Carroll, 1993). The other dimension is visual-object style, recently proposed by Blazhenkova and Kozhevnikov (2010), which reflects one's capacity to process information about visual appearances of objects and their pictorial properties (e.g., shape, color, and texture). It is possible that the ability to use object and space visualization reflect how individuals plan and estimate reach ability in the environment.

The question of interest here is how cognitive styles and motor proficiency interact with age to predict accuracy in reach estimation tasks or, in other words, which factors shape accurate reach representations. As previously mentioned, age has been noted as an indicator of accuracy in reach estimation. It is possible that developmental differences in mental imagery tasks are associated to differences in the development of visual object and spatial styles. It appears that through the elementary school years, there is a gradual improvement in the use of visual information and individual frames of reference (Vasilyeva \& Lourenco, 2012), and perhaps this improvement plays a role in the development of reach estimation accuracy. With motor proficiency and cognitive styles (that are related but not dependent on age), could performance accuracy on such tasks be better understood and predicted? To answer these questions, the variables motor proficiency, cognitive styles, and reach estimation accuracy via motor and visual imagery were measured in seven- to 13-year-old children. A generalized linear mixed model technique was used to account for the variables. This procedure allows for a separation of the possible predictors into specific levels, dissociating age as a random effect, which is a baseline category where other fixed effect predictors (motor proficiency and cognitive styles) are free to interact. In other words, this statistical approach allows 
emergence of other possible predictors that may be covered by age effects. Up to this point, most previous studies have used standard analyses that do not account for the differentiation or hierarchy between age and other parameters.

Research goal. Using GLLM, age was used as a potential predictor of motor proficiency. If successful, GLLM allows assessment of motor development without limiting analyses by age. It was expected that all independent variables would be significant predictors of accuracy in both reach estimation tasks.

\section{METHOD}

\section{Participants}

This convenience sample consisted of 38 volunteers (13 girls, 25 boys) ranging from 7 to 13 years old $(M=9.3, S D=2.1)$ of age (7-year-olds: $n=10$; 8 -year-olds: $n=7$; 9-year-olds: $n=5$; 10 -year-olds: $n=4$; 11-year-olds: $n=2$; 12-year-olds: $n=8$; 13 -year-olds: $n=2$ ). Participants were recruited through emails, flyers, and advertisements on boards in the community surrounding the university where the study was conducted. All children were righthanded, as determined by results of the Lateral Preference Inventory (Coren, 1993). The choice of the age range was based on task feasibility. All parents filled out a questionnaire to ensure that no child had a history of past or present sensorimotor impairment and had normal or optically corrected-tonormal vision. The Institutional Review Board (IRB) for the ethical treatment of human subjects at the University of Texas at Arlington approved the experimental protocol and consent form. All children provided verbal assent after parents signed the consent form.

\section{Measures}

Cognitive styles.-To detect spatial and object imagery styles, the Children's Object-Spatial Imagery Questionnaire (C-OSIQ) (Blazhenkova, Becker, \& Kozhevnikov, 2011) Object Scales (OS) and Spatial Scales (SS) were administered. According to the authors, this questionnaire has been reported to have high predictive, discriminant, and ecological validity. Ecological validity was established by correlating object and spatial subscales with students' ratings of learning preferences for various subjects. For a complete list of ecological validity measures, see Blazhenkova, et al. (2011). Predictive validity was established by correlating object and spatial subscale values with their criterion measures, with convergent validity for object $(r=.42$ and .18$)$ and spatial $(r=.42$ and .34$)$ scales. Values for internal consistency reliability measured by Cronbach's $\alpha$ were .87 for the object scale and .71 for the spatial scale, and values for test-retest reliability were .65 for the object and .71 for the spatial scale. The self-report ques- 
tionnaire was designed to assess individual differences in visual imagery preferences and experiences associated with object and spatial ability in adults. The test consists of scaled (1-5) items targeted to assess object and spatial imagery (15 items each) preferences and experiences, as well as estimates of proficiency in object and spatial image processing, estimates of ability to perform tasks requiring the use of either object or spatial imagery, and ratings of qualitative characteristics. Scores for each item are added to compute the score for each scale, with a minimum score of 15 and a maximum total score of 75. As noted earlier, research indicates that the object scale was moderately correlated with performance on object imagery tasks, while the spatial scale was moderately associated with behavior on spatial imagery tasks.

Reach estimation (motor imagery).- - Actual maximum reach (used for comparison) and imaged reach responses were collected via a short-throw projection system (Sanyo Model PLC-XL50) linked to a computer programmed with Java (Fig. 1a, b). Visual images were systematically projected onto a table surface at midline $\left(90^{\circ}\right)$. The table was constructed on a sliding bracket frame, allowing it to be moved back and forward for adjustment to the participant. Participants sat in an adjustable ergonomic chair fixed and aligned with the midline of the table and projected image midline. The seat height (surface was metal and nondepressive) was set to $105 \%$ of the participant's popliteal height, the distance from the underside of the foot to the underside of the thigh at the knees. The table height was then adjusted to the midpoint between seat height and seated eye height. Table and seatpan positioning were modified from Carello, Grosofsky, Reichel, Solomon, and Turvey (1989) and Choi and Mark (2004). To aid in establishing actual reach limitations for an action with one degree of freedom (described in the next section), a commercial seatbelt system was modified and secured to the back of the chair. The room was darkened with the exception of light from the computer monitor and visual images projected onto a white colored tabletop; reach targets consisted of white, $2 \mathrm{~cm}$ diameter circles. The fixation point was projected at midline approximately $45 \mathrm{~cm}$ from the most distal target.

To begin, participants were systematically positioned in the chair and introduced to the task for determining maximum reach: full extension of the dominant (right) limb and middle finger to slide forward a penny using a one-degree of freedom ( $1 d f$ ) reach (Carello, et al., 1989). A $1 d f$ reach involved a comfortable effort of the hand, forearm, and upper arm acting as a single functional skeletal unit. Based on maximum reach, six imagery targets were randomly programmed, with the third item representing actual reach complemented with three target sites farther and two sites closer touching at the rims. Participants were asked to focus while kin- 

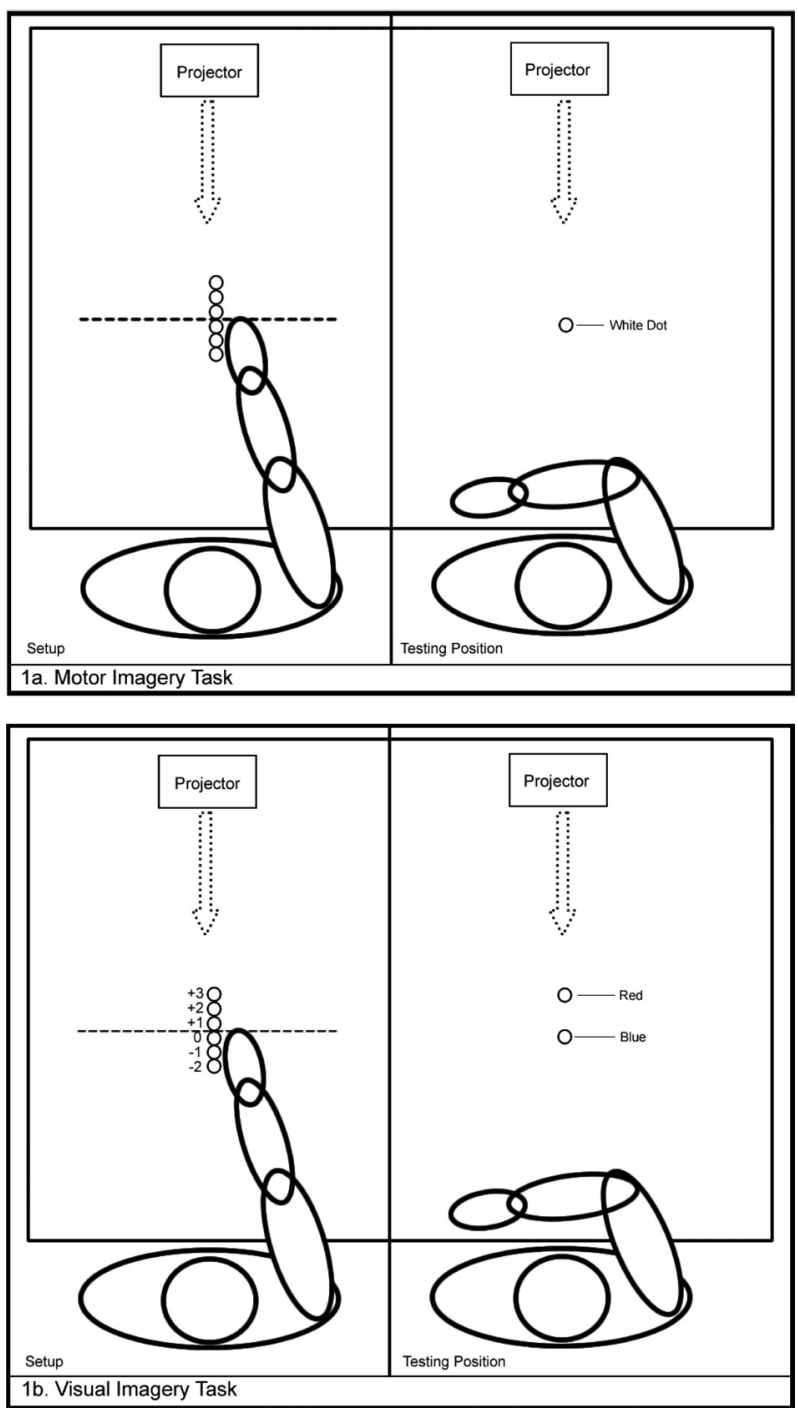

FIG. 1. General experimental setup for motor imagery and visual imagery tasks. 1a. Motor Imagery Task. Setup: Measurement of participant's maximum reach (at dotted line), and dots projected based on participant's maximum reach. Testing Position: Participant estimates whether each projected dot is within reach or not. 1b. Visual Imagery Task. Setup: Measurement of participant's maximum reach (at dotted line), and dots projected based on participant's maximum reach. Testing Position: Participant estimates placement of red target in relation to blue target. 
esthetically "feeling" themselves (first-person perspective) executing the movement with the dominant limb, therefore being more sensitive to the biomechanical constraints of the (motor imagery) task (Johnson, Corballis, \& Gazzaniza, 2001). Participants were asked to make judgments relative to whether a target displayed was within reach ("yes") or not ("no"). Data collection began with a $5 \mathrm{sec}$. "Ready!" signal, immediately followed by a central fixation point lasting 3 sec., at the end of which was a tone. The image of one target appeared immediately thereafter and lasted $1 \mathrm{sec}$. with another tone at the end, which required an immediate (after motor imaging) verbal response. The target presentation was given in random order with three trials at each of the six target locations, totaling 18 trials.

The choice of the imagery tasks using the notion of maximum reach is based on strong evidence that the mechanism subtending the specification of spatial boundaries might essentially depend on the ability to anticipate the consequences of deployable actions (e.g., Coello \& Delevoye-Turrell, 2007). According to this idea, any visual stimulus can automatically evoke a potential motor action, which, regardless of whether the action is subsequently executed or not, maps the spatial stimulus in motor terms. Associated with this visuomotor mapping, a predictive system provides anticipatory information about action outcome (forward model, Wolpert \& Kawato, 1998; Grush, 2004; Choudhury, Charman, Bird, \& Blakemore, 2007). The function of this predictive system is not only to prepare the motor system for execution and to facilitate on-line guidance, but also to provide the self with minimal information on the achievability of actions (Jeannerod, 2006). The distinction between reachable and non-reachable objects has been suggested to rely on this predictive system and exists only by virtue of this potentiality (Coello \& Delevoye-Turrell, 2007).

Reach estimation task (visual imagery).-The basic experimental (projection) setup in regard to table and scaling of the individual to the task was the same as with the motor imagery task. The visual imagery task required participants to estimate object location (red target) relative to an initially cued blue target (Fig. 1b). The blue target was a representation of the individual participant's actual maximum reach (using the method described with the motor imagery task). To facilitate the visual representation of the target, the participants were trained and instructed to use visual imagery.

The task required that the participant focus on the cued blue target, then estimate the location (distance) of the red target in reference to the blue target. The red target $(2 \mathrm{~cm}$ circle) was positioned randomly at one of three $2 \mathrm{~cm}$ circle locations above or two locations below the blue target (Fig. 1b), providing six targets with a range of -2 to +3 . The trial began with a 5 sec. "Ready!" signal, immediately followed by a central fixation 
point lasting $3 \mathrm{sec}$. , at the end of which the participant heard a tone and the blue target appeared for $1 \mathrm{sec}$. Immediately after disappearing, the red target appeared for $1 \mathrm{sec}$, at the end of which was a second tone signaling the participant to respond. Theoretically, visual imagery aided the participant in remembering the relative location of the blue target, which was used to estimate the red target location. For example, the participant would answer " +2 " to estimate that the red target was two targets above the cued (blue) target. Three trials at each of the six target sites were presented randomly (18 trials total). No performance feedback was available to participants. Cordova and Gabbard (2012) have provided a description and report of use of this paradigm using children and young adults.

Motor proficiency $(M P)$. - The Bruininks-Oseretsky Test of Motor Proficiency (BOT-2) was used to determine the participants' overall motor ability (Bruininks \& Bruininks, 2005). This assessment covers the age range of four to 21 years, with a 53-item assessment consisting of eight subtests designed as game-like tasks that measure hand and arm coordination, balance, mobility, and strength. Four motor-area composites (Fine Manual Control, Manual Coordination, Body Coordination, and Strength \& Agility) are examined to create a Total Motor Composite (TMC) score indicating overall motor skills of the individual. Overall, internal consistency defined by subtest reliabilities ranged from the high $.7 \mathrm{~s}$ to the low $.8 \mathrm{~s}$, and the total motor composite reliabilities fell in the mid .9s. Test-retest reliability coefficients ranged from .69 to .80 , whereas interrater reliability coefficients ranged from .92 to .99. Factor analysis confirmed the composite model with CFI $=.95$. Concurrent validity was determined with the Peabody Developmental Motor Scale $(r=.73)$, and discriminant validity was assessed with children with Developmental Coordination Disorder and mild to moderate retardation. There is an accumulation of evidence for the validity of the BOT-2 strongly supported through both empirical and theoretical sources (Düger, Bumin, Uyanika, Aki, \& Kayihan, 1999; Wilson, Kaplan, Crawford, \& Dewey, 2000).

\section{Procedure}

Individual testing involved two sessions of 60 to $90 \mathrm{~min}$. each within a $2 \mathrm{wk}$. period, where the order of the tests was counterbalanced. All testing was conducted in an isolated room. During the first session, participants completed consent forms and a handedness questionnaire. Next, participants were tested with either the reach estimation tasks (MIT/VIT) and the C-OSIQ or the BOT-2. The tasks not completed during the first session were completed during the second session.

Analysis

For object and spatial cognitive styles, the total score was the sum for each subscale based on participants' responses for each one of the 15 items. 
Total motor composite scores using percentile ranks were used for motor proficiency. For the motor imagery task, a score of " 1 " was given to a correct verbal response when the participant responded "yes" when the target was indeed within reach or "no" when in fact the target was beyond reach. For the visual imagery task, a response was counted correct and scored a " 1 " when the participant responded with the correct estimate of the response (red) target from the cued (blue) target; e.g., "+2" when the target was indeed a distance of +2 above the cued target. The data were analyzed using a generalized linear mixed model (GLMM) approach, also known as a multilevel logistic regression model (MLRM). The GLMM is used for modeling data associated with participants clustered into various levels of a higher level variable (e.g., age), where participants across different clusters are measured on a binary outcome (success or failure) variable and one or more predictor variables (Wong \& Mason, 1985). In this case, age was considered as "baseline," a category that allows exploration of how other factors interact with age and predict a given ability within that age group. This approach enables unique relationships to emerge, which might otherwise be obscured by age effects (Pangelinan, Zhang, VanMeter, Clark, Hatfield, \& Haufler, 2011). The appropriate procedure for modeling such data is available in the SAS software as PROC GLIMMIX. The GLMM allows us to assume that the age groups were random and therefore representative of the population under study. With that, age could be dissociated as a random effect from other fixed effect predictors, such as motor proficiency and cognitive styles. The empirical model developed for these data analysis used the following terms:

The dependent variable $y_{i j}\left(i=1,2, \ldots, n_{j}\right.$ and $\left.j=1,2, \ldots, J\right)$ is a binary outcome (success or failure) variable assumed to have a binomial distribution, i.e., $y_{i j} \sim \operatorname{Bin}(n, \pi)$, where $n(=18)$ is the number of trials and $\pi=P\left(y_{i j}=1\right)$ is the probability of success in each trial. The independent (predictor) variables were categorized as such: first, Chronological Age (CA) was regarded as an effect factor in order to account for the effects of a higher-level entity, and considered separate intercepts in the model for each age cluster. These random intercepts measured the differences among the age clusters, controlling for the other predictors in the model. In other words, each child had a "different rank" to start off based on his or her age. Second, motor proficiency (MP) and cognitive styles (OS and SS) were considered participant-level predictor variables (fixed effects), meaning that the effect was specific and linked to the actual data set, and an estimate of the parameter was found for each group. Therefore, the model was established as:

$$
\operatorname{logit}\left(\pi_{i j}\right)=\alpha_{j}+\beta_{1} \mathrm{MP}_{i j}+\beta_{2} \mathrm{OS}_{i j}+\beta_{3} \mathrm{SS}_{i j}+e_{i j}
$$


where the predicted value of $\pi i j$ was expressed as

$$
\hat{\pi}_{i j}=\frac{\exp \left(\alpha_{j}+\beta_{1} \mathrm{MP}_{i j}+\beta_{2} \mathrm{OS}_{i j}+\beta_{3} \mathrm{SS}_{i j}\right)}{1+\exp \left(\alpha_{j}+\beta_{1} \mathrm{MP}_{i j}+\beta_{2} \mathrm{OS}_{i j}+\beta_{3} \mathrm{SS}_{i j}\right)^{\prime}} \text { and } e_{i j} \sim N\left(0, \sigma_{e}^{2}\right)
$$

were independent error terms. Therefore, the logit model (that transforms odds ratios to a scale that ranges from possible negative to possible positive values) in (1) had a linear function at the logit [or, log(Odds Ratio $=\mathrm{OR})]$ scale. Also, $\alpha_{j}$ in (1) represented a unique intercept for age cluster $j(j=1,2, \ldots j)$, and for estimation purposes each $\alpha_{j}$ was a random intercept given by

$$
\alpha_{j}=\alpha+u_{j}
$$

where $\alpha$ was the grand mean of the intercepts and $u_{j}$ was the deviation (or error associated with random intercepts) of each random intercept from the mean. The error terms $u_{j} \sim N\left(0, \sigma_{u}^{2}\right)$ in (2) were independent of the participant-level random errors $\left(e_{i j}\right)$.

The regression model in (1) and (2), together, formed a multilevel logistic regression model (MLRM) with two levels of hierarchy, with Level 1 (children) and Level 2 (age). At Level 1, the outcome was expressed as the sum of an intercept for the participant's age and the product of the participant's potential predictors (motor proficiency, object scale, and spatial scale) and their slope coefficients. At Level 2, the age intercepts were specified as the sum of an overall mean and the random deviations from that mean. A 5\% level of significance was used for all the statistical hypothesis tests, and the models in (1) and (2) were combined to form one model as:

$$
\operatorname{logit}\left(\pi_{i j}\right)=\alpha+\beta_{1} \mathrm{MP}_{i j}+\beta_{2} \mathrm{OS}_{i j}+\beta_{3} \mathrm{SS}_{i j}+u_{j}+e_{i j} \text {. }
$$

\section{RESULTS}

The dependent variables $y_{i j}$ for the motor imagery and visual imagery tasks were not significantly correlated $(r=.38)$; therefore, a separate GLMM analysis was run for each task. All possible models for the motor imagery task were run starting from the null model (intercepts only) and then adding and removing each participant-level predictor (i.e., MP, OS, and SS) and all possible interactions. The most efficient model in GLMM analyses is conventionally selected by comparing fit statistics such as -2Res $\log P L$, where a smaller value is better. For motor imagery, that best model was the one with motor proficiency and object scale as predictors, and for visual imagery the best model was the one with motor proficiency only as the predictor. The intercepts and slope coefficients associated with these two models are shown in Table 1.

Based on values showed in Table 1, the GLMM equation for the motor imagery task was determined as: 
TABLE 1

Estimates of Parameters (Standard Errors) and Other Statistics For Motor IMAgery and Visual IMAgery TASKS

\begin{tabular}{|c|c|c|c|c|}
\hline & \multicolumn{4}{|c|}{ GLIMMIX } \\
\hline & \multicolumn{2}{|c|}{ Motor Imagery } & \multicolumn{2}{|c|}{ Visual Imagery } \\
\hline & Parameter & $S E$ & Parameter & $S E$ \\
\hline \multicolumn{5}{|l|}{ Fixed Effects } \\
\hline Intercept & $2.60^{*}$ & 0.91 & 0.12 & 0.28 \\
\hline Motor proficiency & $0.009^{*}$ & 0.004 & $0.01 \dagger$ & 0.004 \\
\hline Object scale & $-0.03^{*}$ & 0.01 & - & \\
\hline \multicolumn{5}{|l|}{ Random Effect } \\
\hline \multicolumn{5}{|l|}{ Intercepts only } \\
\hline Age $=7$ & $-0.66^{*}$ & 0.27 & $-0.58^{*}$ & 0.22 \\
\hline Age $=8$ & -0.24 & 0.28 & -0.17 & 0.23 \\
\hline Age $=9$ & -0.27 & 0.29 & 0.02 & 0.24 \\
\hline Age $=10$ & 0.23 & 0.33 & $0.47^{+}$ & 0.26 \\
\hline Age $=11$ & 0.51 & 0.37 & 0.19 & 0.28 \\
\hline Age $=12$ & $0.51^{+}$ & 0.29 & -0.04 & 0.22 \\
\hline \multicolumn{5}{|l|}{ Age $=13$} \\
\hline Odds ratio (Motor proficiency) & -0.09 & 0.36 & 0.10 & 0.29 \\
\hline Odds ratio (Object scale) & 1.01 & 0.97 & 1.01 & - \\
\hline Intra-age correlation $(\rho)$ & \multicolumn{2}{|c|}{.07} & \multicolumn{2}{|c|}{.04} \\
\hline Extra dispersion & \multicolumn{2}{|c|}{2.37} & \multicolumn{2}{|c|}{2.14} \\
\hline -2ResLogPL & \multicolumn{2}{|c|}{140.55} & \multicolumn{2}{|c|}{116.00} \\
\hline
\end{tabular}

Note.-GLIMMIX is the SAS procedure for GLMM. †Estimate $\rightarrow p<.01$, *Estimate $\rightarrow$ $p<.05,{ }^{+}$Estimate $\rightarrow p<.10$.

$$
\begin{aligned}
& \operatorname{logit}\left(\hat{\pi}_{i j}\right)=2.60+0.01{ }^{*} \mathrm{MP}_{i j}+(-0.03) * \mathrm{OS}_{i j} \\
& +(-0.66) *(\text { Age }=7)+(-0.24) *(\text { Age }=8)+(-0.27) \\
& *(\text { Age }=9)+(0.23) *(\text { Age }=10)+(0.51) *(\text { Age }=11) \\
& +(0.51) *(\text { Age }=12)+(-0.09) *(\text { Age }=13)
\end{aligned}
$$

where the mean intercept $\alpha=2.60$ was significant $\left(t_{6}=2.87, p=.029\right)$, the slope coefficient $\beta_{1}=.01$ was significant $\left(t_{29}=2.06, p<.05\right)$, and the slope coefficient $\beta_{2}=-.03$ was significant $\left(t_{29}=2.05, p<.05\right)$. The equation shows that MP and OS were significant predictors of success rates at $5 \%$ level of significance, and also indicated that the random intercept $\alpha_{j}=-.66$ associated with the age of seven also displayed significance $\left(t_{35}=-2.49, p<.05\right)$. All the remaining random intercepts were not significant. The GLMM analysis in (4) was also used to estimate the logit function of the predicted 
probability of accuracy in reach estimation in the motor imagery task for $i$ th participant in the $j$ th age group, where $\left(i=1,2, \ldots, n_{j}\right.$ and $\left.j=1,2, \ldots, J\right)$, and values are shown in Table 2.

TABLE 2

Observed and Predicted Probabilities For Motor Imagery and Visual Imagery Tasks

\begin{tabular}{|c|c|c|c|c|c|c|c|c|}
\hline \multirow{2}{*}{ ID } & \multirow{2}{*}{ Age } & \multirow{2}{*}{$\mathrm{MP}$} & \multirow{2}{*}{ SS } & \multirow{2}{*}{ OS } & \multicolumn{2}{|c|}{ Motor Imagery } & \multicolumn{2}{|c|}{ Visual Imagery } \\
\hline & & & & & Observed & Predicted & Observed & Predicted \\
\hline 4 & 7 & 31 & 74 & 64 & 0.67 & 0.59 & 0.56 & 0.48 \\
\hline 8 & 7 & 96 & 47 & 56 & 0.67 & 0.77 & 0.78 & 0.67 \\
\hline 14 & 7 & 84 & 50 & 71 & 0.89 & 0.66 & 0.39 & 0.64 \\
\hline 20 & 7 & 96 & 46 & 49 & 0.72 & 0.80 & 0.83 & 0.67 \\
\hline 24 & 7 & 90 & 50 & 47 & 0.78 & 0.80 & 0.89 & 0.65 \\
\hline 29 & 7 & 95 & 45 & 59 & 0.72 & 0.75 & 0.61 & 0.67 \\
\hline 30 & 7 & 62 & 50 & 54 & 0.50 & 0.72 & 0.22 & 0.57 \\
\hline 36 & 7 & 97 & 51 & 48 & 0.89 & 0.81 & 0.72 & 0.67 \\
\hline 39 & 7 & 35 & 51 & 61 & 0.72 & 0.62 & 0.56 & 0.49 \\
\hline 47 & 7 & 69 & 48 & 54 & 0.56 & 0.73 & 0.33 & 0.59 \\
\hline 7 & 8 & 73 & 41 & 64 & 0.83 & 0.77 & 0.39 & 0.70 \\
\hline 22 & 8 & 89 & 47 & 50 & 0.83 & 0.85 & 0.72 & 0.74 \\
\hline 23 & 8 & 46 & 60 & 60 & 0.94 & 0.74 & 0.89 & 0.62 \\
\hline 35 & 8 & 79 & 42 & 58 & 0.83 & 0.80 & 0.83 & 0.71 \\
\hline 38 & 8 & 92 & 63 & 66 & 0.89 & 0.79 & 0.89 & 0.74 \\
\hline 40 & 8 & 46 & 59 & 53 & 0.61 & 0.78 & 0.50 & 0.62 \\
\hline 43 & 8 & 99 & 50 & 59 & 0.56 & 0.83 & 0.61 & 0.76 \\
\hline 2 & 9 & 73 & 73 & 73 & 0.50 & 0.71 & 0.61 & 0.74 \\
\hline 5 & 9 & 42 & 53 & 67 & 0.50 & 0.69 & 0.56 & 0.66 \\
\hline 48 & 9 & 58 & 44 & 72 & 0.56 & 0.69 & 0.83 & 0.70 \\
\hline 54 & 9 & 76 & 65 & 66 & 1.00 & 0.75 & 0.78 & 0.74 \\
\hline 56 & 9 & 42 & 26 & 52 & 1.00 & 0.77 & 0.72 & 0.66 \\
\hline 3 & 10 & 21 & 43 & 46 & 0.78 & 0.85 & 0.78 & 0.70 \\
\hline 12 & 10 & 84 & 48 & 56 & 1.00 & 0.88 & 0.72 & 0.83 \\
\hline 16 & 10 & 27 & 32 & 45 & 0.83 & 0.86 & 0.72 & 0.72 \\
\hline 33 & 10 & 16 & 58 & 64 & 0.78 & 0.76 & 0.89 & 0.69 \\
\hline 10 & 11 & 42 & 43 & $\begin{array}{l}68 \\
\text { (con }\end{array}$ & $\begin{array}{c}0.83 \\
\text { nued on ne }\end{array}$ & $\begin{array}{l}0.82 \\
\text { bage) }\end{array}$ & 0.72 & 0.69 \\
\hline
\end{tabular}

Note. $-\mathrm{MP}=$ motor proficiency; $\mathrm{SS}=$ spatial scales; $\mathrm{OS}=$ object scales. Observed probabilities for motor imagery and visual imagery were computed by dividing number of successes for each task by number of trials (18), respectively. Predicted probabilities for motor imagery and visual imagery tasks were respectively computed using right hand side (RHS) expressions of (4) and (5) in (6). 
TABLE 2 (CONT'D)

Observed and Predicted Probabilities For Motor Imagery and Visual Imagery Tasks

\begin{tabular}{|c|c|c|c|c|c|c|c|c|}
\hline \multirow{2}{*}{ ID } & \multirow{2}{*}{ Age } & \multirow{2}{*}{$\mathrm{MP}$} & \multirow{2}{*}{ SS } & \multirow{2}{*}{ OS } & \multicolumn{2}{|c|}{ Motor Imagery } & \multicolumn{2}{|c|}{ Visual Imagery } \\
\hline & & & & & Observed & Predicted & Observed & Predicted \\
\hline 25 & 11 & 38 & 39 & 61 & 0.94 & 0.85 & 0.72 & 0.68 \\
\hline 6 & 12 & 54 & 37 & 59 & 1.00 & 0.87 & 0.67 & 0.68 \\
\hline 9 & 12 & 38 & 48 & 56 & 0.78 & 0.86 & 0.44 & 0.63 \\
\hline 13 & 12 & 66 & 61 & 70 & 0.78 & 0.85 & 0.78 & 0.71 \\
\hline 19 & 12 & 35 & 52 & 60 & 0.83 & 0.85 & 0.44 & 0.62 \\
\hline 41 & 12 & 16 & 51 & 58 & 0.94 & 0.83 & 0.44 & 0.57 \\
\hline 44 & 12 & 38 & 66 & 69 & 0.61 & 0.81 & 0.83 & 0.63 \\
\hline 49 & 12 & 86 & 52 & 64 & 1.00 & 0.89 & 0.83 & 0.75 \\
\hline 53 & 12 & 84 & 45 & 67 & 1.00 & 0.88 & 0.89 & 0.75 \\
\hline 11 & 13 & 58 & 58 & 63 & 0.61 & 0.77 & 0.61 & 0.71 \\
\hline 55 & 13 & 73 & 43 & 48 & 1.00 & 0.86 & 0.89 & 0.75 \\
\hline
\end{tabular}

Note. $-\mathrm{MP}=$ motor proficiency; $\mathrm{SS}=$ spatial scales; $\mathrm{OS}=$ object scales. Observed probabilities for motor imagery and visual imagery were computed by dividing number of successes for each task by number of trials (18), respectively. Predicted probabilities for motor imagery and visual imagery tasks were respectively computed using right hand side (RHS) expressions of (4) and (5) in (6).

The GLMM for the visual imagery task was determined using estimates from Table 1 as:

$$
\begin{aligned}
& \operatorname{logit}\left(\hat{\pi}_{i j}\right)=0.13+0.01{ }^{*} \mathrm{MP}_{i j}+(-0.58) *(\text { Age }=7) \\
& +(-0.17) *(\text { Age }=8)+(0.02)^{*}(\text { Age }=9)+(0.47) \\
& *(\text { Age }=10)+(0.19) *(\text { Age }=11)+(-0.04) \\
& *(\text { Age }=12)+(0.10)^{*}(\text { Age }=13)
\end{aligned}
$$

where the mean intercept $\alpha=0.13$ was not significant $\left(t_{6}=0.45, p=.67\right)$ but the slope coefficient $\beta_{1}=.01$ was significant $\left(t_{30}=3.22, p<.05\right)$. The equation shows that motor proficiency was a significant predictor of accuracy rates. The random intercept $\alpha_{i}=-.58$ associated with the age of $7 \mathrm{yr}$. was significant $\left(t_{36}=-2.67, p<.05\right)$, and all the remaining random intercepts were not significant. The GLMM in (5) was used to estimate the logit function of the predicted probability of accuracy in reach estimation in the visual imagery task of $i$ th participant in the $j$ th age group, where $\left(i=1,2, \ldots, n_{j}\right.$ and $\left.j=1,2, \ldots, J\right)$, and values are shown in Table 2 . The models to determine the predict probabilities $\left(\hat{\boldsymbol{\pi}}_{i j}\right)$ for both motor imagery and visual imagery tasks, based on (3), were determined as: 


$$
\hat{\pi}_{i j}=\frac{\exp \left(\alpha_{j}+\beta_{1} \mathrm{MP}_{i j}+\beta_{2} \mathrm{OS}_{i j}+\beta_{3} \mathrm{SS}_{i j}\right)}{1+\exp \left(\alpha_{j}+\beta_{1} \mathrm{MP}_{i j}+\beta_{2} \mathrm{OS}_{i j}+\beta_{3} \mathrm{SS}_{i j}\right)}
$$

where $\alpha_{j}+\beta_{1} \mathrm{MP}_{i j}+\beta_{2} \mathrm{OS}_{i j}+\beta_{3} \mathrm{SS}_{i j}$ in the numerator and denominator of (6) was replaced by the right-hand side expression of (4) and (5) to obtain the predicted probabilities for the motor imagery and visual imagery tasks, respectively (described in Table 2).

In summary, the GLMM models in (4) and (5) can be used for predicting each participant's value that can be subsequently used in (6) to obtain predicted probability of accuracy at reach estimation, thus allowing inferences from group level to individual level. Overall, the results indicated that 7-year-olds had the only significant random intercept among the age random intercepts used in the predictive model for both motor imagery and visual imagery tasks. Motor proficiency and the cognitive style measured by object scale were the only significant fixed factors that predicted reach estimation accuracy in the motor imagery task, whereas motor proficiency was the only significant fixed effect predicting reach estimation accuracy in the visual imagery task.

\section{Discussion}

The purpose of this study was to apply a generalized linear mixed model (GLMM) technique that can account for the assumption that motor development is age-related but not age-dependent by analyzing how age as a higher level variable and other factors of a child's developmental status (motor proficiency and cognitive styles) interact to affect accuracy of reach estimation tasks. The rationale behind the purpose arose from the notion that age can often obscure other factors that could reflect developmental levels such as motor proficiency and cognitive styles (object and spatial scales). It was expected that all factors would be statistically significant predictors of accuracy in both reach estimation tasks. Results indicated that 7-year-olds had the only significant random intercept among the age random intercepts used in the predictive model, for both motor imagery and visual imagery tasks. Motor proficiency was the only significant fixed effect for both tasks, and the cognitive style measured by the Object Scale significantly predicted reach estimation accuracy in motor imagery task.

First, it is worth noting that the intercept for the 7-year-old age group was the only significant intercept among all age intercepts. The age of seven is considered a social milestone, and traditionally it has been established as the period that reflects "readiness" of the system, marking the end of early childhood years that provided a foundation for further cognition and learning. Not surprisingly, the ages of six and seven mark the beginning of formal instruction and standardized schooling (Dacey \& Travers, 2006). Physically, 
children at ages six and seven have a repertoire of gross motor skills and start sharpening fine-motor skills, as seen in the refinement of handwriting abilities (Gabbard, 2011), for example. Cognitively, the time between ages six and eight is one of tremendous change, as children start using logic and reason for independent problem solving, entering Piaget's concrete operational stage, in which thought is logical, flexible, and organized in its application to concrete information (Berk, 1999). With all those changes, it is not unreasonable to argue that the 7-year-old intercept was a significant predictor of accuracy in both tasks while none of the others were.

More specifically to the present study, the age of seven years is a marker for the development of mental imagery abilities, most specifically motor imagery. Research using both subjective and objective measures of motor imagery indicated that children as young as five years of age have the ability to imagine movements (Funk et al., 2005), and this ability appears to be still emerging at seven years of age (Molina, et al., 2008; Frick, et al., 2009). Other studies have indicated clear age differences with significant improvements occurring at seven and eight years of age (Caeyenberghs, et al., 2009), which is possibly related to the notion that with 7-year-olds motor imagery emerges from their own sensorimotor sensation (Molina, et al., 2008). Thus, motor imagery would be centered on the sensation of the action, i.e., on the capacity to feel the proprioceptive consequences of one's own action. Another explanation for the enhanced ability of 7-yearolds is provided by Gabbard (2009), who suggests that underlying the use of each of the tasks is the notion that children can engage in mental imagery processes and have the cognitive ability, as well as attentiveness, to understand and complete the task.

Remarkably, the most interesting finding was that motor proficiency was an overall significant predictor of accuracy in both motor and visual imagery tasks. Motor proficiency is likely to be a factor that interacts with age, shaping how children represent, plan, and act on the environment. In some cases, age can obscure motor proficiency effects on a child's developmental progress. It is well known that motor imagery capacity depends directly on real motor activity of children (Caeyenberghs, et al., 2009), with the relationship between motor imagery and motor skills getting stronger with age while visual imagery does not seem to be related to motor skills at any age. In the present study, motor proficiency was a significant predictor of accuracy for both motor imagery and visual imagery reach estimation tasks. While the tasks required use of motor and visual imagery separately, both involved reach estimation, and it is possible that motor proficiency plays a role with accuracy prediction in reach estimation tasks. For example, Jarraya, Chtourou, Souissi, and Chamari (2011) found that soccer players were more accurate in a perception of distance task when 
compared to sedentary individuals, and there is some evidence that golfers are more accurate than others in estimating distances on grass (e.g., Durgin \& Li, 2011).

Cognitive styles, on the other hand, were not as important in reach estimation accuracy when compared to motor proficiency. With the exception of the object scale predicting accuracy in the motor imagery task, no other significant predictors were found. This finding is interesting because the term "spatial" hints at a possible higher association with motor imagery in comparison to "object." While spatial style reflects a number of related subcomponents (e.g., spatial visualization, spatial relations) that relate to how individuals deal with information presented in space, object styles commonly refers to the ability to process information about visual appearances of objects and their properties (e.g., shape, color, and texture). A possible explanation for the lack of relationship with spatial styles and motor imagery is the fact that the type of spatial evaluation required by that task might not be associated with distances to (and from) one's body schema (Caçola, et al., 2004). Speculatively, in children, the preference for the object style may have added metric parameters that were useful when estimating reach via motor imagery.

Overall, the relationship between cognitive styles and reach estimation can be inferred from the notion that cognitive abilities have been established as key components in action processing (Gallese, Rochat, Cossu, \& Sinigaglia, 2009; Al-Yahya, Dawes, Smith, Dennis, Howells, \& Cockburn, 2011; Cassenti, 2011; Nip, Green, \& Marx, 2011), and the processing used for distance judgments requires individuals to extract visual information from different viewpoints and convert that information into coordinates. However, a methodological consideration could explain the lack of major significant findings: the evaluation of object and spatial styles were "perceived," and may not reflect the individuals' actual (real) ability, as was the case for motor proficiency. According to Blazhenkova, et al. (2011), although the development of cognitive styles and that of corresponding ability are closely linked, their developmental trajectories are somewhat different, with cognitive styles developing more gradually and smoothly.

The statistical model used here was developed to analyze the specific purpose of the present study, but the model can be expanded and used by other researchers who wish to understand the intertwining of age and other developmental factors in childhood. The major difference between the generalized linear mixed model and other models that are more commonly used in the field is the fact that this model allows other factors to "emerge" instead of being obscured by age effects. Typical statistical models do not use a hierarchy of predictors, which can be beneficial for the un- 
derstanding of age and the relationship of age with other parameters that are influenced by age. The model shows probabilities that are close to the actual numbers and, notably, has the ability to utilize age as a potential predictor for performance but not to limit the analysis by age. For example, when using a design where age is an independent variable and accuracy in motor/visual imagery tasks is the dependent variable, the analysis is limited by assuming that emergent differences are due to age itself. In other words, analyzing age as a predictor creates the assumption that age is comparable to other factors, and in many cases age explains variance in a dependent variable that could have been shared by other factors. With this model, age is taken into consideration from a hierarchical perspective (it is intrinsic to each child), along with other abilities and skills that can be (and, in this case, are) important parameters influencing performance in a task. From another perspective, it is clear that the model has limitations; e.g., it may not be the best choice when the purpose of the study is to investigate group effects or parameters that are not hierarchical.

Overall, these findings indicate that the generalized linear mixed model is a suitable technique to analyze how age and other parameters of development interact to shape behavioral parameters. The results revealed that motor proficiency was the most prominent predictor of accuracy in reach estimation tasks viewed from both motor imagery and visual imagery perspectives. The authors can only speculate as to those whose abilities they evaluated, but the results suggested that motor proficiency, especially after the age of seven years, could be a more unique predictor of a child's motor and visual imagery abilities. It is obvious that motor proficiency depends on age, but using an appropriate analysis in this study helped to determine a more specific contribution of motor proficiency in reach estimation accuracy. Of course, it is not possible to discount other confounding factors that may have influenced reach estimation accuracy, such as attention, experience, confidence, and spatial movement awareness (Coello \& Delevoye-Turrell, 2007).

\section{Limitations and Conclusions}

Some limitations of this study include the small sample size within age groups, as well as the fact that the participants volunteered for the tasks, which could have increased their motivation. In addition, it is important to point out that the assessment of cognitive styles and motor proficiency, as well as motor and visual imagery, may not fully represent the extent of the children's abilities for each respective construct. In addition, using the percentile ranks for the assessment of motor proficiency may have biased the results. Fortunately, the type of analysis used here accounts for the sample size issue, since the random intercepts are related to individuals and not considered representative of a group. This study was the first to show a 
unique perspective on how other developmental factors (in this case, motor proficiency and cognitive styles) can transcend age in shaping accuracy of reach estimation tasks. It is likely that age is sometimes used as a predictor for motor development because children's experiences tend to be similar in certain age groups, especially with children from similar cultural backgrounds. However, it is important to reinforce the use of other predictors (related to age or not) that could give important insights in a child's performance. In this study, specifically, motor proficiency was more prominent than age in predicting reach estimation accuracy. Using age alone could potentially limit the role that other developmental predictors play in predicting accuracy of distance estimation tasks. Future studies are needed to further explore the relationships between age and other developmental factors, and how those can better predict a child's behavior.

\section{REFERENCES}

Adolph, K. E., TAmis-LeMonda, C. S., \& KarasiK, L. B. (2010) Cinderella indeed-a commentary on Iverson's 'Developing language in a developing body: the relationship between motor development and language development.' Journal of Child Language, 37, 269-273. DOI: 10.1017/S030500090999047X

Al-Yahya, E., Dawes, H., Smith, L., Dennis, A., Howells, K., \& Cockburn, J. (2011) Cognitive motor interference while walking: a systematic review and metaanalysis. Neuroscience \& Biobehavioral Reviews, 35, 715-728. DOI: 10.1016/j.neubiorev.2010.08.008

Ausburn, L. J., \& Ausburn, F. B. (1978) Cognitive styles: some information and implications for instructional design. Educational Communication and Technology, 26, 337354.

Blazhenkova, O., Becker, M., \& Kozhevnikov, M. (2011) Object-spatial imagery and verbal cognitive styles in children and adolescents: developmental trajectories in relation to ability. Learning and Individual Differences, 21(3), 281-287. DOI: 10.1016/j. lindif.2010.11.012

BlazhenKova, O., \& Kozhevnikov, M. (2010) Visual-object ability: a new dimension of non-verbal intelligence. Cognition, 117, 276-301. DOI: 10.1016/j.cognition.2010. 08.021

BERK, L. E. (1999) Infants, children, and adolescents. (3rd ed.) Needham Heights, MA: Allyn \& Bacon.

BruininKs, R. H., \& BRUININKs, B. D. (2005) Bruininks-Oseretsky Test of Motor Proficiency. (2nd ed.) Minneapolis, MN: Pearson.

Caçola, P., Spessato, B., \& Gabbard, C. (2014) An age-related view of the role of object and spatial cognitive styles in distance estimation. Journal of Cognitive Psychology, 26(2), 147-156. DOI: 10.1080/20445911.2013.876028

Caeyenberghs, K., Tsoupas, J., Wilson, P. H., \& Smits-Engelsman, B. C. M. (2009) Motor imagery in primary school children. Developmental Neuropsychology, 34(1), 103121. DOI: $10.1080 / 87565640802499183$

Campos, J. J., Anderson, D. I., Barbu-Roth, M. A., Hubbard, E. M., Hertenstein, M. J., \& Witherington, D. (2000) Travel broadens the mind. Infancy, 1(2), 149-219.

Carello, C., Grosofsky, A., Reichel, F. D., Solomon, H. Y., \& Turvey, M. T. (1989) Visually perceiving what is reachable. Ecological Psychology, 1, 27-54. 
CArroll, J. (1993) Human cognitive abilities: a survey of factor-analytical studies. New York: Cambridge Univer. Press.

CASSENTI, D. (2011) The intrinsic link between motor behavior and temporal cognition. New Ideas in Psychology, 29, 72-79. DOI: 10.1016/j.newideapsych.2010.03.011

CHOI, H. J., \& MARK, L. S. (2004) Scaling affordances for human reach actions. Human Movement Science, 23, 785-806. DOI: 10.1016/j.humov.2004.08.004

Choudhury, S., Charman, T., Bird, V., \& Blakemore, S. J. (2007) Development of action representation during adolescence. Neuropsychologia, 45(2), 255-262.

Clark, J. E., \& Metcalfe, J. M. (2002) The mountain of motor development: a metaphor. In J. E. Clark \& J. H. Humphrey (Eds.), Motor development: research and reviews. Vol. 2. Reston, VA: National Association for Sport and Physical Education. Pp. 163-190.

Coello, Y., \& Delevoye-Turrell, Y. (2007) Embodiment, spatial categorization and action. Consciousness and Cognition, 16, 667-683. DOI: 10.1016/j.concog.2007.07.003

Cordova, A., \& GabBard, C. (2012) Children's use of allocentric cues in visually- and memory-guided reach space. International Journal of Behavior Development, 36(1), 1-6. DOI: $10.1177 / 0165025411426681$

CoRen, S. (1993) The Lateral Preference Inventory for measurement of handedness, footedness, eyedness, and earedness: norms for young adults. Bulletin of the Psychonomic Society, 31(1), 1-3.

DACEY, J. S., \& Travers, J. F. (2006) Human development across the lifespan. (6th ed.) New York: McGraw-Hill.

Decety, J., \& GrĖZes, J. (1999) Neural mechanisms subserving the perception of human actions. Trends in Cognitive Sciences, 3, 172-178. DOI: 10.1016/S1364-6613(99)013121

Düger, T., Bumin, G., UyaniKa, M., AKI, E., \& KaYiHAN, H. (1999) The assessment of Bruininks-Osteretsky test of motor proficiency in children. Pediatric Rehabilitation, 3 , 125-131.

Durgin, F. H., \& Li, Z. (2011) Perceptual scale expansion: an efficient angular coding strategy for locomotor space. Attention, Perception, \& Psychophysics, 73, 1856-1870. DOI: $10.3758 / \mathrm{s} 13414-011-0143-5$

Frick, A., DAum, M. M., Wilson, M., \& WiLKenING, F. (2009) Effects of action on children's and adults' mental imagery. Journal of Experimental Child Psychology, 104(1), 34-51. DOI: $10.1016 /$ j.jecp.2009.01.003

Funk, M., Brugger, P., \& Wilkening, F. (2005) Motor processes in children's imagery: the case of mental rotation of hands. Developmental Science, 8(5), 402-408. DOI: $10.1111 /$ j.1467-7687.2005.00428.x

GABBARD, C. (2009) Studying action representation in children via motor imagery. Brain and Cognition, 71(3), 234-239. DOI: 10.1016/j.bandc.2009.08.011

Gabbard, C., Cordova, A., \& Ammar, D. (2007) Estimation of reach in peripersonal and extrapersonal space: a developmental view. Developmental Neuropsychology, 32(3), 749-756. DOI: $10.1080 / 87565640701539451$

GABBARD, C. P. (2011) Lifelong motor development. (6th ed.) San Antonio, TX: Pearson.

Gallahue, D. L., Ozmun, J. C., \& Goodway, J. D. (2012) Understanding motor development. (7th ed.) Columbus, $\mathrm{OH}$ : McGraw-Hill Education.

Gallese, V., Rochat, M., Cossu, G., \& Sinigaglia, C. (2009) Motor cognition and its role in the phylogeny and ontogeny of action understanding. Developmental Psychology, 45, 103-113. DOI: $10.1037 /$ a00 14436 
Grush, R. (2004) The emulation theory of representation: motor control, imagery, and perception. Behavioral and Brain Sciences, 27(3), 377-396. DOI: 10.1017/S0140525X 04000093

Haywood, K. M., \& GetchelL, N. (2009) Life span motor development. (5th ed.) Champaign, IL: Human Kinetics.

Jarraya, M., Chtourou, H., Souissi, N., \& Chamari, K. (2011) Effect of aerobic physical exercise and training status on the perceived egocentric distances. BIO Web of Conferences, 1(00042). DOI: 10.1051/ bioconf/ 20110100042

JEANNEROD, M. (2006) Motor cognition: what actions tell the self. Oxford, UK: Oxford Univer. Press.

Johnson, S., Corballis, P., \& GazZAniga, M. (2001) Within grasp but out of reach: evidence for a double dissociation between imagined hand and arm movements in the left cerebral hemisphere. Neuropsychologia, 39, 36-50. DOI: 10.1016/S00283932(00)00 096-8

KossLyn, S. M. (1999) If neuroimaging is the answer, what is the question? Philosophical Transactions of the Royal Society, Biological Sciences, 354(1387), 1283-1294. DOI: 10. 1098 / rstb.1999.0479

Molina, M., Tijus, C., \& Jouen, F. (2008) The emergence of motor imagery in children. Journal of Experimental Child Psychology, 99(3), 196-209.

Nip, I. S. B., GreEn, J. R., \& MARX, D. B. (2011) The coemergence of cognition, language, and speech motor control in early development: a longitudinal correlation study. Journal of Communication Disorders, 44, 149-160. DOI: 10.1016/j.jcomdis.2010.08.002

Oliver, B., GabBard, C., Caçola, P., \& Bobbio, T. (2012) Examining the age-related ability to maintain visual images. International Journal of Mind, Brain, \& Cognition, 3(1), 59-72.

Pangelinan, M. M., Zhang, G., VanMeter, J. W., Clark, J. E., Hatfield, B. D., \& Haufler, A. J. (2011) Beyond age and gender: relationships between cortical and subcortical brain volume and cognitive-motor abilities in school-age children. NeuroImage, 54(4), 3093-3100. DOI: 10.1016/j.neuroimage.2010.11.021

Rieser, J. J., GARING, A. E., \& YounG, M. F. (1994) Imagery, action, and young children's spatial orientation: it's not being there that counts, it's what one has in mind. Child Development, 65, 1262-1278.

Roberton, M. A. (1982) Describing 'stages' within and across motor tasks. In J. A. S. Kelso \& J. E. Clark (Eds.), Psychology of motor behavior and sport-1979. Champaign, IL: Human Kinetics. Pp. 269-279.

VASILYEVA, M., \& Lourenco, S. F. (2012) Development of spatial cognition. WIREs Cognitive Science, 3, 349-362. DOI: 10.1002/wcs.1171

Wilson, B. N., Kaplan, B. J., CRAWFord, S. G., \& Dewey, D. (2000) Interrater reliability of the Bruininks-Osteretsky Test of Motor Proficiency-Long Form. Adapted Physical Activity Quarterly, 17, 95-110.

WolPERT, D. M., \& KAWATO, M. (1998) Multiple paired forward and inverse models for motor control. Neural Networks, 11(7-8), 1317-1329. DOI: 10.1016/S0893-6080(98) 00066-5

WONG, G. Y., \& MASON, W. M. (1985) The hierarchical logistic regression model for multilevel analysis. Journal of the American Statistical Association, 80(391), 513-524.

Accepted August 11, 2014. 